\title{
Ulrich Niederer*
}

\section{Zwei Seiten einer Münze: Die Kooperative Speicherbibliothek Schweiz und die Kooperative Print-Archivierung}

https://doi.org/10.1515/bfp-2017-0045

Zusammenfassung: In den letzten zehn Jahren wurden in der Schweiz zwei große, kooperative Projekte zur Aufbewahrung von gedrucktem Material in der Schweiz realisiert, die sich mit ihren komplementären Prinzipien und den unterschiedlichen Verfahren ergänzen. Dargestellt wird zunächst die Kooperative Speicherbibliothek. Der Artikel erklärt, was sie ist und wem sie dient und zeichnet nach, wie es dazu gekommen ist. Im zweiten Teil stellt er die Kooperative Print-Archivierung vor, die im Gegensatz zur zentralisierenden Aufbewahrung auf dezentrale Archivierung und damit auf die Selbstverpflichtung und Eigenverantwortung bei den einzelnen Bibliotheken setzt.

Schlüsselwörter: Außenlager; Kooperation; Print-Bestände

Two Sides of a Coin: the Cooperative Storage Library Switzerland and the Cooperative Archiving of Printed Serials

\begin{abstract}
Two comprehensive, cooperative projects of printed material storage have been realised in Switzerland during the last ten years. They complement each other in their principles and their diverse procedures. The Cooperative Storage Library Switzerland (CSLS) will be presented first. The article informs about the use of the storage library, who participates, whom it serves, and how it was realised. The second part of the article will be devoted to the Cooperative Archiving of Printed Serials (CAPS). Whereas the CSLS is a place for centralised storage for the participating libraries, the CAPS is based on the principle of de-centralised storage, of self-obligation and responsibility of the participating libraries.
\end{abstract}

Keywords: Off-site storage; cooperation; printed material
Wie viele Exemplare eines Titels sollen in Bibliotheken aufbewahrt werden? Seit dem Aufkommen von kooperativen Magazinierungseinrichtungen nach dem Zweiten Weltkrieg stellt sich diese Frage immer wieder, und erst recht seit dem digital turn. Verschiedene Initiativen und Projekte in mehreren Ländern zeigen unterschiedliche Möglichkeiten und auch Grenzen: Waren sie zunächst auf Zeitschriften fokussiert, so rücken vermehrt auch Monografienbestände in den Blick.

Der hier folgende Artikel soll zwei Initiativen aus der Schweiz vorstellen, die komplementär zueinander entstanden sind. Im ersten Abschnitt wird die Kooperative Speicherbibliothek Schweiz (KSBS) vorgestellt: Was ist sie? Wem dient sie? Wie kam sie zustande und wie funktioniert das Ganze heute, eineinhalb Jahre nach Betriebsaufnahme? Der zweite Teil behandelt die Kooperative Printarchivierung (KPA), die seit etwa zehn Jahren die garantierte, verteilte Aufbewahrung von Zeitschriftentiteln verfolgt. Schließlich wird aufgezeigt, wie die Komplementarität aussieht und wie die Überschneidungen zwischen den beiden Projekten diskutiert werden.

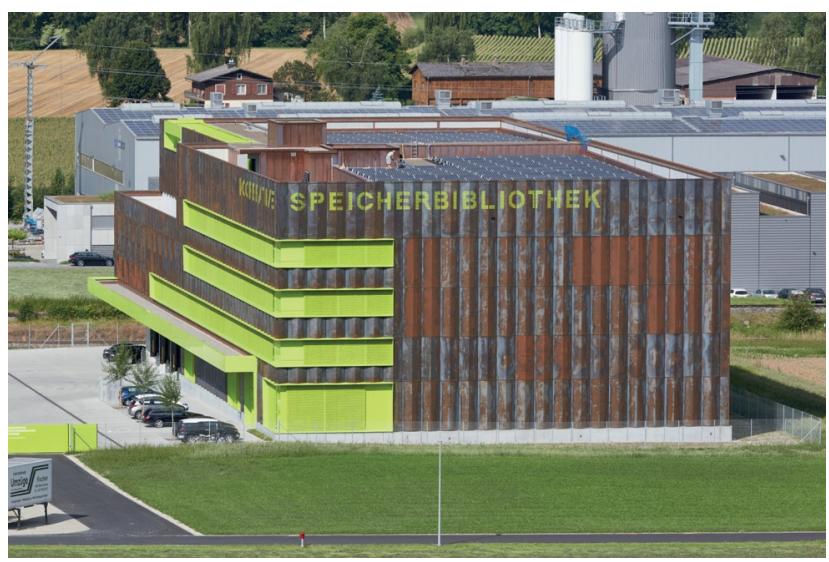

Abb. 1: Die Kooperative Speicherbibliothek Schweiz von außen. Juli 2016 (Foto: U. Niederer)

*Kontaktperson: Ulrich Niederer, ulrich_niederer@bluewin.ch 


\section{Die Kooperative Speicherbibliothek Schweiz (KSBS)}

Die KSBS, die Speicherbibliothek, wie sie kurz genannt wird, dient sechs Bibliotheken aus der deutschsprachigen Schweiz als Magazin, als Außenlager: der Bibliothek der Universität Basel, der Zentral- und Hochschulbibliothek Luzern, der Bibliothek der Universität St. Gallen, der Zentralbibliothek Solothurn, den Bibliotheken der Universität Zürich und der Zentralbibliothek Zürich. Sie steht in Büron, einem Dorf im Kanton Luzern mit sehr guter Verkehrsanbindung. Die Fahrdistanz beträgt für die heute teilnehmenden Bibliotheken zwischen einer halben (Luzern) und anderthalb Stunden (St. Gallen). Die KSBS bietet Platz in ihrem ersten Lagermodul für ca. 3,1 Mio. Bände, angemeldet sind bisher von allen sechs Bibliotheken ca. 2,8 Mio. Bände. Auf dem Grundstück können weitere Module mit einer Gesamtkapazität von bis zu 14 Mio. Bänden erstellt werden.

Die Speicherbibliothek ist als Hochregal-Lager ausgelegt, die Bücher werden in Behältern gelagert, die von Robotern, d.h. Regalbediengeräten, und einer BehälterFörderanlage zwischen Lager und Verwaltungsbereich transportiert werden. Im Verwaltungsbereich, an sogenannten Kommissionierungsplätzen werden die Bücher in die Behälter abgefüllt oder zur Erfüllung von Bestellungen entnommen. Die Hochregale sind 14 Meter hoch, das Lager ist 70 Meter lang und enthält 6 Korridore (Gassen), in denen die Regalbediengeräte fahren. Auf jeder Seite einer Gasse sind die Behälter doppeltief gelagert. Ein Regalbediengerät kann zwei Behälter übereinander aufnehmen.

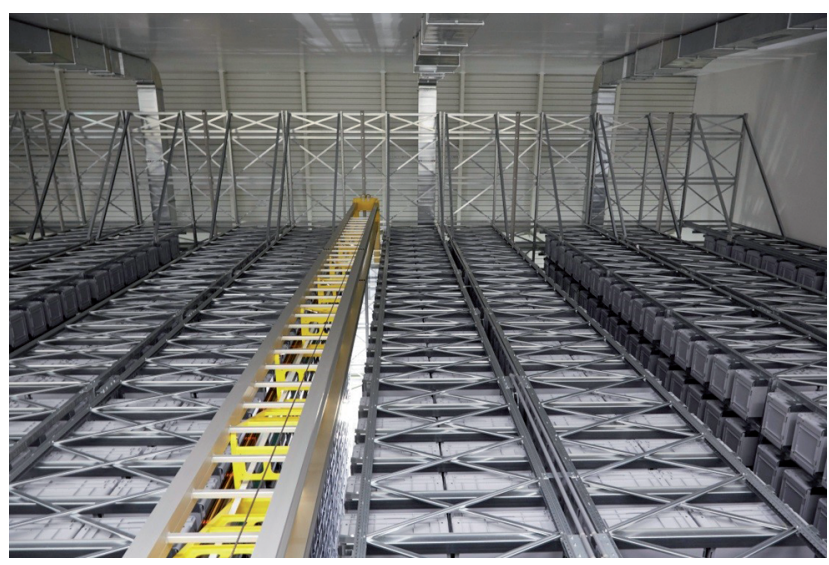

Abb. 2: Die Hochregale mit doppeltief eingelagerten Behältern auf jeder Seite einer Gasse: das gelbe, leiterartige Element ist Teil eines Regalbediengerätes (Foto: U. Niederer)

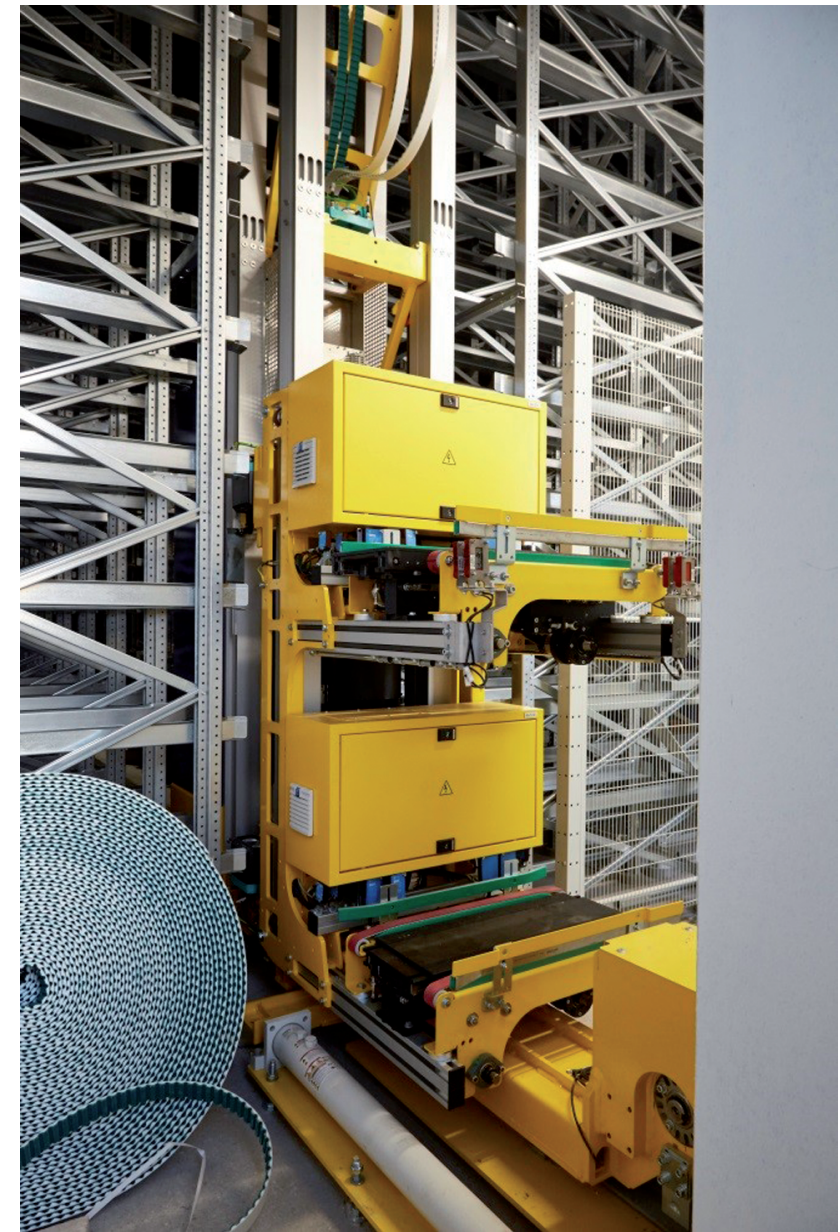

Abb. 3: Das Regalbediengerät mit den übereinanderliegenden Lastaufnahmemitteln (Foto: U. Niederer)

Die Behälter haben das Standardmaß von 60 x $40 \mathrm{~cm}$. Es werden zwei Höhen verwendet: 25 und $35 \mathrm{~cm}$. Die Behälter enthalten im Durchschnitt 29 Bände. Sie können längs oder quer befüllt werden, Großformate werden auch liegend eingelagert. Die Behälter dürfen maximal $60 \mathrm{~kg}$ schwer sein. Das Gewicht wie auch die Höhe der Bücher in den Behältern (sie dürfen unter keinen Umständen über den Rand des Behälters hinausragen) werden an den Kommissionierungsplätzen kontrolliert.

Im Lagerbereich arbeiten keine Personen, daher kann der Sauerstoffgehalt im Lagerbereich so gesenkt werden, dass kein Feuer entstehen kann: eine sehr wirksame und kostengünstige Art der Brandverhütung. Die Speicherbibliothek konnte nach intensiver Vorbereitung und einer kurzen Bauzeit von 18 Monaten im Februar 2016 in Betrieb genommen werden. Inzwischen sind knapp 2 Mio. Bände eingelagert. 


\subsection{Entwicklung einer Idee}

Wie kommt man auf die Idee einer solch hochtechnologischen Einrichtung für ein Bibliotheksmagazin? Einerseits: es gibt in Europa und Nordamerika bereits einige automatisierte Hochregallager in Bibliotheken. Wahrscheinlich die älteste, die Schlesische Bibliothek, steht in Katowice. Bekannter ist der nördliche Teil der Nationalbibliothek Norwegens in Mo i Rana, die vor kurzem ihrem ersten automatisierten Modul ein zweites hinzugefügt hat. Mehrere ,off-site storage facilities' in Nordamerika sind seit dem Harvard Depository (1986) als Hochregallager ausgebildet, allerdings sind sie nicht automatisiert, sondern sie werden mit bemannten Fahrgeräten bedient und können deshalb nicht inertisiert, d.h. mit Sauerstoffreduktion betrieben werden. Ähnlich funktioniert das CTLes, das Centre Technique du Livre de l'éducation supérieure bei Paris sowie die Book Storage Facility des Oxford University Library System. Die einzige andere Bibliothek, die bisher beides, ein automatisiertes Hochregallager und die Sauerstoffreduktion, realisiert hat, ist die British Library mit ihren ,additional storage buildings' an ihrem zweiten Standort in Boston Spa, in der Nähe der Stadt York. ${ }^{1}$

Andererseits: Zwischen 2005 und 2007 hatte das Hochbauamt des Kantons zusammen mit der Zentral- und Hochschulbibliothek Luzern eine detaillierte Evaluation des besten Lagertyps durchgeführt, damals noch beschränkt auf die Bedürfnisse der Luzerner Bibliothek. Ziel der Evaluation war, die konservatorisch und ökonomisch beste Art der Lagerung zu eruieren. Es war damals gerade deutlich geworden, dass eine Aufbewahrung aller ihrer Bestände an einem Ort und in der Stadt nie mehr möglich sein würde. Zudem legte die Entwicklung des Haupthauses es nahe, die vorhandenen Magazinflächen in Freihandbereiche und Publikumsräume umzuwandeln. Die Evaluationsgruppe stand unter der Leitung des Hochbauamtes und umfasste neben drei Mitgliedern aus der Bibliothek auch einen externen Logistik-Berater (Ralog Engineering AG, Zofingen).

Um die Vergleichbarkeit der verschiedenen Modelle zu gewährleisten, wurde angestrebt, die Kosten so genau und umfassend wie möglich zu berechnen; sowohl die Investitions- und Betriebskosten als auch die Kapital- und Amortisationskosten mit einzubeziehen und in der Größe ,Kosten pro Band pro Jahr' festzuhalten. Vorgegeben waren: Platz für 1,5 Mio. Bände, modulare Erweiterbarkeit, kon-

1 Ein ausführlichere Darstellung der Luzerner Evaluation sowie der verschiedenen Hochregallager-Typen findet sich in Niederer (2016). servatorische Bedingungen (18 Grad, \pm 2 Grad, $45 \%$ rel. Luftfeuchtigkeit, $\pm 5 \%$ ), Fahrzeit vom Haupthaus in der Stadt Luzern nicht mehr als eine halbe Stunde. Untersucht wurden in einem ersten Durchgang die Modelle

- Traditionelles Lager mit feststehenden Büchergestellen,

- Lager mit Fahrregalen,

- Hochregal-Lager mit Bedienung durch bemannte Fahrgeräte (nach dem ersten solcher Lager, dem Harvard Depository, oft auch als Harvard-type bezeichnet),

- Hochregal-Lager mit automatisierter Bedienung und Sauerstoffreduktion.

Schon bei diesem ersten Durchgang wurde deutlich, dass vier Kostenfaktoren eine zentrale Rolle spielen:

- Land- oder Grundstückskosten,

- Lohnkosten und Arbeitsvorschriften,

- Brandschutzkosten,

- Stahlkosten.

Die ersten beiden sind regional oder auch national bestimmt, können also durchaus variabel sein. In der Schweiz etwa sind die Grundstückskosten hoch, deshalb war der Vergleich des Flächenbedarfs von hohem Interesse: Bei gleicher Anzahl Bände und gleicher Bauhöhe hat das traditionelle Lager den größten Flächenbedarf. Er wurde mit dem Faktor 1 gesetzt. Dagegen weist das FahrregalLager einen Faktor von 0.68 auf, das Harvard-type-Lager einen Faktor von 0.85 und das automatisierte Lager einen Faktor von 0.32 .

Auch die Lohnkosten resp. Arbeitsvorschriften können sehr unterschiedlich sein: Während zum Beispiel in den USA die bemannten Fahrgeräte, die sogenannten order pickers, in den Harvard-type-Lagern von Aushilfskräften oder Studierenden bedient werden können, sind in der Schweiz dafür nur gelernte Fachleute mit spezieller Führerprüfung zugelassen.

Der dritte und vierte Faktor hingegen sind ziemlich unabhängig von lokalen Gegebenheiten. Der Brandschutz ist für die verschiedenen Lagertypen gesondert zu betrachten: Während in traditionellen Lagern Sprinkler durchaus Standard mit vertretbaren Kosten darstellen, so sind sie in Hochregal-Lagern sowohl in Installation als auch im Unterhalt ausgesprochen aufwändig. Zudem bleibt das Wasser in den Behältern stehen, was doppelt ungünstig ist: Einerseits ist nicht gewährleistet, dass genügend Wasser den Brandherd erreicht, und andererseits ist Wasser an Büchern schon schlimm genug - Bücher im Wasser sind verheerend. Dagegen ist die Inertisierung, die Sauerstoffreduktion zwar nicht kostenfrei, aber doch unschlagbar günstig, aber sie ist nur möglich in 

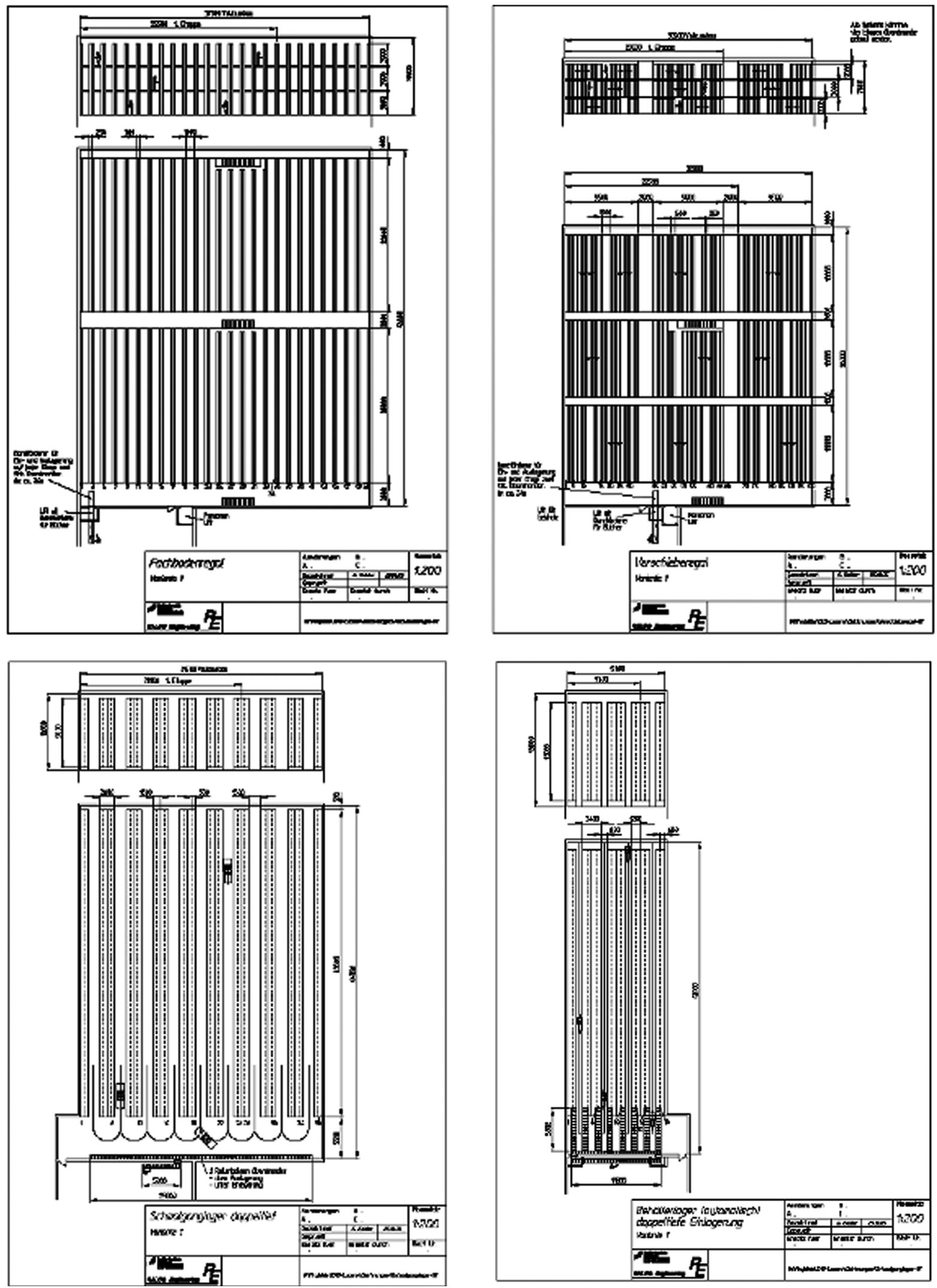

Abb. 4: Lager-Typen und schematische Planzeichnungen des Flächenbedarfs. 4a: Fachbodenregal, 4b: Verschieberegal, 4c: Schmalganglager, 4d: Behälterlager (vollautomatisch) doppeltief-A (Planzeichnungen: Ralog Engineering AG, Zofingen, Schweiz) 

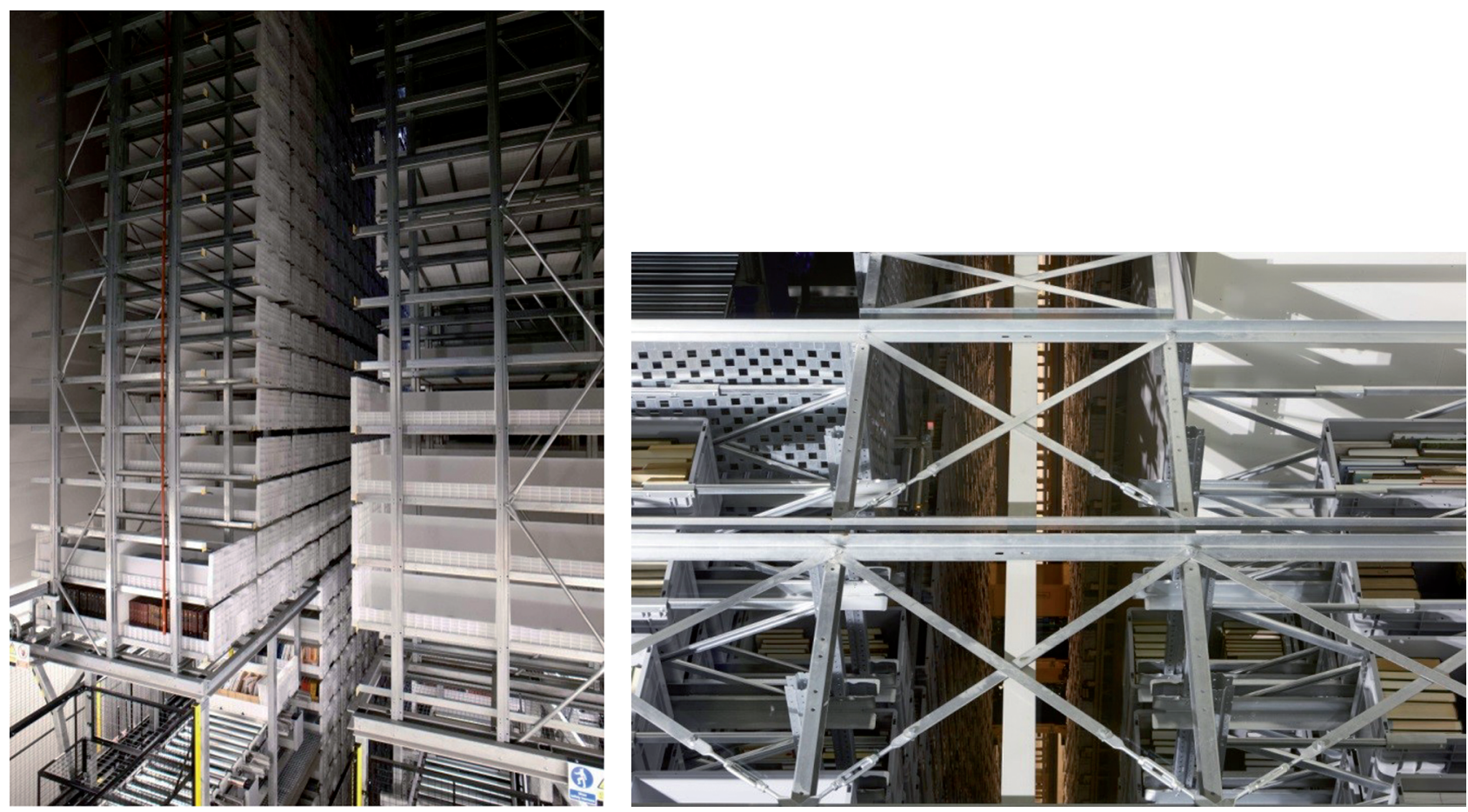

Abb. 5: Die Behälter liegen auf Stahlwinkeln auf, unabhängig von der Größe der Behälter. Hier Beispiele aus dem Additional Storage Building der British Library (weiße Behälter, linkes Bild) und aus der Speicherbibliothek (graue Behälter, rechtes Bild) (Fotos: U. Niederer)

einem Lagerbereich, in dem sich kein Personal regelmäßig und über längere Zeit aufhält. Der Sauerstoff wird von ca. $21 \%$ (Umgebung Höhe Meeresspiegel) auf rund 13,5\% reduziert, das entspricht einer Höhe von ca. 4000 müM. Bei 13,5 \% kann kein Feuer entstehen oder gar bestehen. Die Reduktion geschieht mithilfe von Stickstoff, der vor Ort produziert und in den Lagerbereich eingeblasen wird.

Die Stahlkosten werden durch die Notwendigkeit von Tablaren beeinflusst: Während bei den ersten drei Lagertypen Tablare notwendig sind, braucht das automatisierte Behälterlager nur Stahlwinkel von $5 \mathrm{~cm}$ Breite, auf denen die Behälter aufliegen.

Wenn die ganzen Stahlwinkel großzügig gegen die Tablare gerechnet werden, ergibt sich eine Ersparnis von rund $14000 \mathrm{~m}^{2}$ Stahl.

Diese Resultate führten zu einer zweiten Runde der Evaluation, in der das traditionelle Lager mit feststehenden Regalen mit dem automatisierten Behälter-Lager verglichen wurde. Auf Geheiß der Politik wurde zudem das Modell Outsourcing miteinbezogen: Der gesamte Bestand solle einem Logistik-Dienstleister-Betrieb zur Lagerung nach den geltenden Bedingungen übergeben werden. Der Dienstleister liefere bestellte Bücher ebenfalls nach vereinbarten Bedingungen. Schließlich wurde, sozusagen als reality check, auch der bestehende Zustand, die zufällige Anmietung von Außenlagerflächen jeweils bei Bedarf in der Stadt, in den finanziellen Vergleich integriert. Für alle Modelle wurde ein detailliertes, konkretes Projekt ausgearbeitet: Für das traditionelle Lager wurde der Umbau eines bestehenden Lagergebäudes geplant, für das automatisierte Lager ein Neubau entwickelt, für das Outsourcing-Modell fand eine echte GATT/WTO-Ausschreibung statt und der Status Quo wurde auf die vorgegebenen Mengen hochgerechnet.

Das Resultat fiel wünschenswert deutlich aus: In der Gesamtwertung lag an erster Stelle das automatisierte Hochregal-Lager mit 100 Punkten, an zweiter Stelle das traditionelle Lager mit 91 Punkten und an dritter Stelle das Outsourcing-Modell mit 85 Punkten. Etwas anders sah das Resultat aus, wenn es nur von der Kostenseite her betrachtet wurde: Hier schnitten das automatisierte Hochregal-Lager und die Outsourcing-Variante am besten ab, das traditionelle Lager entpuppte sich als deutlich teurer, aber doch ebenso deutlich günstiger als das Modell des Status Quo, das sich als unschlagbar teuer erwies! In Verhältniszahlen ausgedrückt verhielten sich die Varianten wie 100 für die günstigsten zu $119 \mathrm{zu}$ 137. Ein klarer Beweis dafür, dass sich das planvolle Vorgehen gegenüber dem bloßen Reagieren lohnt.

Der Vergleich zwischen den beiden Modellen automatisiertes Hochregal-Lager und Outsourcing ergab einerseits vergleichbar hohe Kosten, wenn auch bei entgegengesetzter Struktur: Hohe Investitionskosten und niedrige wiederkehrende Betriebskosten für den Neubau des automatisierten Hochregal-Lagers gegen niedrige Investitionskosten, aber hohe wiederkehrende Betriebskosten für die 
Outsourcing-Variante. Zumindest die niedrigen Investitionskosten machten diese Variante attraktiv für den Kanton. Für die Arbeitsgruppe und insbesondere für die Bibliothek bot die Outsourcing-Variante zu wenig langfristige Sicherheit. Gerade die Aufbewahrung ist ein Geschäft mit sehr langer Dauer und große Bestandsmengen können nicht leicht kurzfristig umgelagert werden (etwa bei Vertragskündigung wegen Nichterfüllung der konservatorischen Bedingungen). Was allerdings die Outsourcing-Variante endgültig aus dem Spiel nahm, war die Berechnung des Synergiepotentials für eine größere Anlage: Während sich beim Outsourcing keine erkennbare Synergie bei größeren Mengen zeigte, sanken die Kosten pro Band pro Jahr bei einem Neubau für die doppelte Menge auf $80 \%$, bei einem Gebäude für die dreifache Menge auf $70 \%$. Das überzeugte die politischen Behörden endgültig: Sie brachen die Outsourcing-Ausschreibung formell ab und luden stattdessen die Träger der interessierten Bibliotheken, die sich inzwischen gemeldet hatten, ebenso formell zur Mitarbeit ein.

\subsection{Die Organisation der Zusammenarbeit}

2009 begann die Zusammenarbeit: Zunächst erarbeiteten acht Bibliotheken die rechtliche Formen der neuen Institution Speicherbibliothek. Keiner der Träger der beteiligten Bibliotheken wollte selbst investieren oder die Institution aufbauen und den anderen vermieten. Deswegen wurde die Selbständigkeit der Speicherbibliothek notwendig, ebenso das Prinzip der vollen Kostenvergütung, denn sie musste selbsttragend sein, und das bedeutet, dass sie die entstehenden Kosten vollständig von den beteiligten Bibliotheken erstattet haben muss. Das Finden der rechtlichen Form allerdings brauchte länger. Schließlich wurde entschieden, dass das Eigentum am Gebäude und der Betrieb rechtlich, finanziell und organisatorisch zu trennen sei. Als Eigner des Grundstücks und des Gebäudes wurde eine Aktiengesellschaft mit nicht gewinnorientierter Ausrichtung gegründet, der die beteiligten Institutionen resp. deren Träger angehören können (aber nicht müssen). Heute sind der Kanton Luzern, die Universität Basel und die Zentralbibliothek Zürich (die die Form einer selbständigen Stiftung hat) Aktionäre - das Aktionariat zeigt schon die Vielfalt der Trägerformen der Bibliotheken. Die AG Speicherbibliothek nahm das für den konkreten Bau notwendige Geld am Finanzmarkt auf, begleitete den $\mathrm{Bau}^{2}$ als Bauherrin und

2 Für Details zum Bau, etwa dem Prinzip der passiven Klimatisierung, und zur Ausstattung vgl. Tschirren und Niederer (2015), Tschirren und Niederer (2016) sowie Tschirren, Märki und Niederer (2016). vermietet das fertige Gebäude an den Verein Kooperative Speicherbibliothek Schweiz. Diesem Verein, der als Organ für den Betrieb dient, gehören die beteiligten Bibliotheken an. Nachdem von den anfänglich acht Bibliotheken drei aus sehr unterschiedlichen Gründen ihre Mitarbeit aufgeben mussten, bildeten fünf der eingangs erwähnten Bibliotheken den Kreis der Gründungsmitglieder, die sechste, die Bibliothek der Universität St. Gallen, stößt im Herbst 2017 als erstes Neumitglied hinzu.

Das beleuchtet zugleich das hohe Interesse, das die Speicherbibliothek auf allen Ebenen an der Erweiterbarkeit hat: Das Grundstück bietet Fläche für weitere dreieinhalb Lager-Module der heutigen Größe, der Verwaltungs- und Haustechnikbereich kann leicht auf Erweiterungen angepasst werden, und die Entwicklung von neuen Dienstleistungen ist stets im Fokus. Andererseits ist das Interesse an der Speicherbibliothek groß, die Besuche aus der ganzen Schweiz kommen in dichter Folge, und auch aus dem Ausland melden sich viele Besucher an, um sich in die Überlegungen zu diesem kooperativen Unterfangen einführen zu lassen.

\subsection{Funktionale und operative Prinzipien}

Zu diesen Überlegungen gehören auch einige bibliothekarischen Charakters. Etwa die Vorbedingung, dass kein Buch ungereinigt ins Lager gebracht wird: Entweder reinigen die Bibliotheken die abzuliefernden Bestände selbst oder sie nehmen die Reinigung als (zu bezahlende) Dienstleistung der Speicherbibliothek in Anspruch. Ebenso ist festgelegt, dass kein Buch ohne Katalogisat und ohne Barcode in die Speicherbibliothek kommt. Barcode, Autor- und Titelangaben, Benutzungsbedingungen gehören zu den Stamm- oder Kerndaten, die aus den Bibliothekssystemen der teilnehmenden Bibliotheken an das Lagerverwaltungssystem geliefert werden. Das Lagerverwaltungssystem steuert die Regalbediengeräte, kontrolliert die Verbindung zwischen Buch, Behälter und Lagerplatz. Ein Buch hat einen festen, unveränderlichen Platz in einem Behälter, die Behälter hingegen werden dynamisch gelagert. Die Sicherung des Lagerverwaltungssystems erfolgt redundant, um eine möglichst hohe Datensicherheit zu gewährleisten. Eine händische Suche im Behälter-Hochregallager ist - ganz abgesehen vom geringen Sauerstoffgehalt - nicht vorstellbar ...

Mit der Datenübermittlungs-Kommunikation zwischen Bibliothekssystem und Lagerverwaltungssystem ist zum einen die durchgehende Prozess-Kontrolle möglich: Schon bei der Ersteinlagerung kann resp. muss kontrolliert werden, ob Barcode, Kerndaten und physisches Exemplar übereinstimmen. Das wiederholt sich bei jeder Aus- und Rück- 
lagerung. Zum anderen merken Benutzende nichts von der Speicherbibliothek: Sie bestellen in ihrem Bibliothekssystem und die Speicherbibliothek ist für sie wie ein anderes Außenlager, der Lagerort braucht sie nicht zu kümmern. Wenn die Lieferung physisch erfolgt (vor allem bei Monografien), so wird sie über den schon länger bestehenden Kurierdienst des Informationsverbundes Deutschschweizer Hochschulbibliotheken abgewickelt. Die Speicherbibliothek ist als zusätzlicher Standort integriert und dient überdies als zentraler Hub: Zum Kurierbereich in der Speicherbibliothek hat der Kurierdienst Tag und Nacht Zutritt.

\subsection{Dedoublierung und kollektiver Bestand}

Eine der wichtigsten bibliothekarischen Überlegungen betrifft die Dedoublierung. Die Bibliotheken können individuellen Bestand einlagern, aber es gibt auch einen kollektiven Bestand. Ihm gehören Zeitschriftentitel an, die die Bibliotheken vorher dedoubliert haben. Das bedeutet, dass in den beteiligten Bibliotheken vor der Ablieferung die Zeitschriftentitel auf mehrfaches Vorhandensein überprüft werden. Ist mehr als ein Exemplar vorhanden, wird das beste Exemplar eingelagert. Die anderen Exemplare können entsorgt werden, das eingelagerte Exemplar geht in das Eigentum des Vereins resp. der Speicherbibliothek und damit in das Eigentum aller beteiligten Bibliotheken über. Die für den kollektiven Bestand vorgesehenen Titel stellen etwa ein Drittel des angemeldeten Bestandes von 2,8 Mio. Bänden dar, ca. 1 Mio. Bände. Die Dedoublierung weist ein sorgfältig geschätztes Potential von $30 \%$ auf, das Raumspar-Potential beträgt also etwa 300000 Bände oder rund 10000 Laufmeter. Damit wurde die Dedoublierung zum unschlagbar wirksamen Argument, vor allem bei den vorgesetzten Instanzen der Trägerschaften.

Die Durchführung der Dedoublierung erwies sich als ziemlich komplex und aufwändig. Zuerst wurde in den einzelnen Bibliotheken das Korpus der Zeitschriften, die für den kollektiven Bestand zur Verfügung stehen sollten, festgelegt. Diese Titel- und Holdingsangaben der Korpora aus den Katalogen wurden in eine speziell entwickelte Datenbank eingespielt, die den Vergleich und das Online-Arbeiten an diesem Vergleich erlauben sollte. Diese Datenbank machte aufgrund der Holdingsangaben Vorschläge für das vollständigste Exemplar und gab gleichzeitig Hinweise auf fehlende Teile resp. Jahrgänge. Dabei war zuvor festgelegt worden, dass für die naturwissenschaftlichen und die medizinischen Titel der Bestand der Öffentlichen Bibliothek der Universität Basel und für die geistes- und sozialwissenschaftlichen Titel der Bestand der Zentralbibliothek Zürich als Default-Bestände gelten sollten.
Die Vorschläge der Datenbank wurden in den einzelnen Bibliotheken am Bestand direkt überprüft und die zur Überführung in den kollektiven Bestand vorgeschlagenen Titel zumindest stichprobenartig auf Vollständigkeit abgefragt. In den Katalogen wurden die Hinweise zur Zugehörigkeit eines Titels zum kollektiven Bestand eingearbeitet. Diese Hinweise gehören zu den Kerndaten, die die Bibliothekssysteme an das Lagerverwaltungssystem übermitteln, da es zu den bibliothekarischen Prinzipien gehört, dass die Zeitschriften des kollektiven Bestands die Speicherbibliothek physisch nicht mehr verlassen. Sie stehen nur noch für Kopienbestellungen zur Verfügung.

Mit dem kollektiven Bestand realisieren die beteiligten Bibliotheken gleichzeitig zwei gewichtige Vorteile: Sie gewinnen Platz, nicht nur in der eigenen Heim-Bibliothek, sondern auch in der Speicherbibliothek (wo Lagerplatz kostet ...), und sie haben die größtmögliche Sicherheit der Aufbewahrung der abgegebenen Titel in gedruckter Form.

\section{Die Kooperative Print-Archivierung (KPA)}

Diese Sicherheit ist auch das Ziel des zweiten Projekts, das hier vorgestellt werden soll: der kooperativen Archivierung von gedruckten Zeitschriften, kurz KPA. Das Projekt zielt auf ausländische wissenschaftliche Zeitschriften und bezweckt die veröffentlichte Sicherstellung der Aufbewahrung des letzten Exemplars dieser Titel durch die explizite Verteilung der Verantwortlichkeit für die Aufbewahrung auf die teilnehmenden Bibliotheken.

Das Projekt nahm seinen Ausgang in der KUB, der Konferenz der Universitätsbibliotheken der Schweiz. Die KUB versammelt die großen akademischen Bibliotheken aller Landesteile, d. h. auch aller Sprachregionen sowie die Nationalbibliothek. 2006 erhielt sie Unterstützung für ein Projekt zur Langzeitarchivierung von elektronischen Ressourcen. Sie beschloss, parallel dazu eine Arbeitsgruppe einzusetzen, die abzuklären hatte, wie verhindert werden könne, dass durch die Zunahme der E-only-Lizenzierungen von Zeitschriften-Paketen und der zusätzlichen Sicherheit durch die Langzeitarchivierung massenhaft und sozusagen unkontrolliert die Print-Versionen abbestellt würden. Dahinter stand die gemeinsame Überzeugung, dass es zwar ökonomisch interessant und sehr platzsparend sein könne, auf gedruckte zugunsten der elektronischen Ausgaben zu verzichten, dass aber doch ein einziges gedrucktes Exemplar in der Schweiz erhalten bleiben müsse. Die Arbeitsgruppe schlug ein vergleichsweise einfaches, pragmatisches Vorgehen vor: 
- möglichst viele der großen Universitätsbibliotheken miteinbeziehen, aber auch spezialisierte Fachbibliotheken (etwa Bibliotheken großer Universitätsinstitute) zulassen,

- auf die fachlichen Schwerpunkte der Bibliotheken resp. ihrer Träger, der Universitäten, setzen, da hier am leichtesten Kontinuität in der Art und Tiefe der Sammlung erwartet werden könne,

- die Verantwortung für die Bewahrung der gedruckten Bestände auf der gemeinsam beschlossenen Grundlage den einzelnen Bibliotheken in der Form von Selbstverpflichtungen zuweisen,

- diese Verantwortlichkeiten im Schweizerischen Zeitschriften-Portal SZP, das durch die Nationalbibliothek administriert wird, einzutragen, damit auf der Ebene des Zeitschriftentitels klar werden kann, ob ein PrintExemplar schon verpflichtend aufbewahrt wird oder nicht.

Dazu wurden für die Bibliotheken drei Rollen definiert:

1. Bibliothek ohne Archivierungsverpflichtung: Sie übernimmt keine Aufbewahrungspflicht, aber kontrolliert vor der definitiven Abbestellung eines ZeitschriftenTitels, ob dieser Titel von einer anderen Bibliothek als „Archivexemplar“ behandelt wird. Falls ja, steht der Abbestellung nichts im Weg. Falls nein, ist zu klären, ob der Titel noch mehrfach vorhanden ist. Dann stünde der Abbestellung ebenfalls nichts im Weg. Falls es sich aber um das letzte Exemplar handelt, soll sie die Bibliothekscommunity oder die Fachzentralen-Bibliothek, in deren Gebiet der Titel fällt, auf ihre Abbestellungsabsicht aufmerksam machen, sodass eine andere Bibliothek den vorhandenen Bestand oder sogar ein Print-Abonnement übernehmen könnte.

2. Bibliothek mit Archivierungsverpflichtung: Sie übernimmt die Aufbewahrungspflicht und möglichst die Fortsetzung des Print-Abonnements für einen oder mehrere einzelne Titel und trägt diese Verpflichtung im SZP ein.

3. Bibliothek mit Verpflichtung als Fachzentralen: Sie übernimmt die Aufbewahrungspflicht und möglichst die Fortsetzung der Print-Abonnemente für die Titel eines ganzen Fachgebietes.

Schließlich wurden drei wesentliche Dokumente erarbeitet: Das erste hält die Grundlagen fest, das zweite ist eine Liste der Bibliotheken, die als Fachzentralen agieren. Sie enthält auch eine Auflistung der Fachgebiete und nennt dazu die Bibliotheken, die zuständig sind. Das dritte schließlich stellt die Ausführungsbestimmungen zusammen: Wie genau geht man bei der Übernahme einer Ver- pflichtung vor, wie wird der Eintrag im SZP erstellt, was ist bei einer Abbestellung zu beachten etc. Die Dokumente wurden in der KUB diskutiert, im November 2006 verabschiedet und zum 1. Januar 2007 in Kraft gesetzt. ${ }^{3}$ Vor allem die Liste der Fachzentrale wurde ergänzt und korrigiert, die letzte Version stammt vom November 2010.

Seither haben viele Bibliotheken mit Archiv- oder Fachzentralen-Verpflichtung daran gearbeitet, die Titel, für die sie zuständig sind, im Schweizerischen Zeitschriften-Portal SZP als Archivexemplare zu kennzeichnen und wiederum zumindest in Stichproben auf Vollständigkeit zu kontrollieren. Es hat sich zudem gezeigt, dass das Projekt sowohl Stärken als auch Schwächen hat. Zu den Stärken gehört zweifellos die gemeinsame, geteilte Überzeugung, dass es sinnvoll oder gar notwendig ist, ein Print-Exemplar eines Zeitschriftentitels aufzubewahren, die Selbst-Verpflichtung, das pragmatische Verfahren, die Haupt-Orientierung nach Fächern und Fachgebieten. Andererseits gehört zu den Schwächen die gering ausgebildete organisatorische Struktur oder das Fehlen von finanzieller Unterstützung, die eine kontinuierliche Begleitung und vielleicht sogar eine (teilzeitliche) Geschäftsführung einzurichten erlaubt hätte, wie es etwa das ähnlich ausgerichtete englische Projekt UK Research Reserve eindrücklich vormacht.

\subsection{Entwicklungen seit 2007 und Komplementaritäten oder Überschneidungen mit der Speicherbibliothek}

Die Zeitschriftenlandschaft hat sich in den letzten zehn Jahren sehr verändert, der digital turn hat sich spürbar verstärkt und den elektronischen Zugang in den Vordergrund gestellt. Die Lizenzierungs- und Abonnementsmodelle haben sich entsprechend gewandelt. Das beeinflusst die großen Fragen, die von den Bibliotheksverantwortlichen an das KPA-Projekt formuliert werden: Ist es noch notwendig, Print-Abonnemente zu halten, wenn sie immer teurer werden und die Lizenzmodelle so deutlich auf e-only zielen? Und was ist mit den medizinischen Zeitschriften, wenn sich bisher die wichtigen Medizin-Bibliotheken nicht am Projekt KPA beteiligt haben? Diese Bibliotheken haben bereits keine Print-Bestände mehr ... Und schließlich: Ist die Sicherung der Inhalte und des Zugriffs, die mit der garantierten Aufbewahrung des einen Print-Exemplars gewährleistet

3 http://www.kub-cbu.ch/projekte-projets/kooperative-print-archi vierung-cooperation-archivage-des-per-imp/. 
werden kann, inzwischen nicht mit ausgereiften Modellen wie Portico und Lockss gewährleistet?

Es wird auch bezweifelt, dass Bibliotheken langfristige Garantien zur Aufbewahrung von umfangreichen PrintBeständen abgeben können. Magazin-Raum wird vielerorts in Raum für Benutzende umgewandelt. Was, wenn der Träger Forderungen hat, die den Garantien von KPA zuwiderlaufen?

Hier wird oft die Speicherbibliothek ins Spiel gebracht. Denn sie bietet nur durch ihre Anlage die sichere, langfristige Aufbewahrung von Material, insbesondere des kollektiven Bestands. Es wäre auch eine Rolle, die der Speicherbibliothek tatsächlich liegt! Solange sie die Bestände aus den Bibliotheken erhält, die Vereinsmitglieder sind, ist alles ganz einfach. Was aber, wenn Bestände aufbewahrt werden sollen, die nicht von Mitgliedsbibliotheken kommen? Wer bezahlt dann dafür? Zur Erinnerung: Die Speicherbibliothek muss selbsttragend sein, sie hat - so das bisherige Geschäftsmodell - keine anderen Mittel als die, mit denen die Bibliotheken ihr die Dienstleistungen entgelten. Deswegen kann sie heute keine Bestände von Nichtmitgliedsbibliotheken aufnehmen. Aber sie wird zusammen mit den Gremien der schweizerischen Bibliotheken wie der KUB für derartige Anfragen bald Lösungen finden müssen. Es hat bereits eine große Universitäts-Bibliothek, die sich nicht am KPA-Projekt beteiligt, die Speicherbibliothek angefragt, ob sie einen großen Zeitschriftenbestand geschenkt übernehmen wolle. Die Zeitschriften, die aus bisher nicht abgedeckten Fachgebieten stammen, müssten sonst entsorgt werden.

$\mathrm{Zu}$ guter Letzt stellen sich Fragen dieser Art auch über die Landesgrenzen hinaus: Ist es notwendig, die Sicherung von Print in jedem Land - und sei es noch so klein - zu planen und zu realisieren? Wir müssen wohl eher versuchen, auch international explizit zu kooperieren, sich nicht stillschweigend auf die Anderen zu verlassen, um so das Potential zu nutzen, das bereits in verschiedenen anderen Länderinitiativen steckt. ${ }^{4}$
Für die Schweiz jedenfalls stellen die Kooperative Sprint-Archivierung und die Kooperative Speicherbibliothek tatsächlich die zwei Seiten einer Münze dar, die der Währung „Print“ angehört und als Wert „Kooperation“ hat. Wie weit der Geltungsbereich dieser Währung noch reicht, was man damit bezahlen kann oder mit anderen Worten ausgedrückt: Was man dafür erhält, ist vielversprechend, muss aber immer wieder neu definiert werden!

\section{Literatur}

Niederer, Ulrich (2016): Hochregallager - ein neuer Weg der Magazinierung. Die Kooperative Speicherbibliothek Schweiz. Eine Luzerner Fallgeschichte mit Exkursen. In: Hauke, Petra; Werner, Klaus Ulrich (Hrsg.): Praxishandbuch Bibliotheksbau: PlanungGestaltung - Betrieb. Berlin; Boston: De Gruyter, 345-59.

Tschirren, Daniel; Märki, Mike; Niederer, Ulrich (2016): Die Kooperative Speicherbibliothek im Härtetest. In: b.i.t. online, (4) 340-44.

Tschirren, Daniel; Niederer, Ulrich (2015): Die Kooperative Speicherbibliothek Schweiz: eine Erfolgsgeschichte interkantonaler Zusammenarbeit? In: b.i.t. online, (6) 509-12.

Tschirren, Daniel; Niederer, Ulrich (2016): Die Kooperative Speicherbibliothek Schweiz nimmt den Betrieb auf. In: b.i.t. online, (1) 74-77.

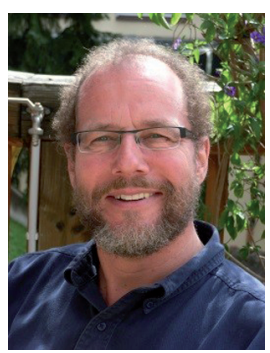

Ulrich Niederer

ehemals Zentral- und Hochschulbibliothek Luzern

Sempacherstrasse 10

$\mathrm{CH}-6002$ Luzern

Schweiz

ulrich_niederer@bluewin.ch

4 Vgl. dazu EPICo, European Print InitiativesCollaboration, eine Gruppe von Repräsentanten verschiedener Projekte zur Aufbewahrung gedruckter Ressourcen: http://www.varastokirjasto.fi/epico/. 